\title{
ON CONSTANT-SIGN PERIODIC SOLUTIONS IN MODELLING THE SPREAD OF INTERDEPENDENT EPIDEMICS
}

\author{
RAVI P. AGARWAL ${ }^{M 1}$, DONAL O'REGAN ${ }^{2}$ and PATRICIA J. Y. WONG ${ }^{3}$
}

(Received 4 April, 2005; revised 18 November, 2005)

\begin{abstract}
We consider the following model that describes the spread of $n$ types of epidemics which are interdependent on each other:

$$
u_{i}(t)=\int_{t-\tau}^{t} g_{i}(t, s) f_{i}\left(s, u_{1}(s), u_{2}(s), \ldots, u_{n}(s)\right) d s, \quad t \in \mathbb{B}, 1 \leq i \leq n .
$$

Our aim is to establish criteria such that the above system has one or multiple constant-sign periodic solutions $\left(u_{1}, u_{2}, \ldots, u_{n}\right)$, that is, for each $1 \leq i \leq n, u_{i}$ is periodic and $\theta_{i} u_{i} \geq 0$ where $\theta_{i} \in\{1,-1\}$ is fixed. Examples are also included to illustrate the results obtained.

2000 Mathematics subject classification: $45 \mathrm{G} 10,47 \mathrm{H} 10$.

Keywords and phrases: periodic solutions, integral equations, epidemics, fixed point theorems.
\end{abstract}

\section{Introduction}

In this paper we shall consider the following system of integral equations that describes the spread of $n$ types of epidemics which are interdependent on each other:

$$
u_{i}(t)=\int_{t-\tau}^{t} g_{i}(t, s) f_{i}\left(s, u_{1}(s), u_{2}(s), \ldots, u_{n}(s)\right) d s, \quad t \in \mathbb{R}, 1 \leq i \leq n .
$$

Here, for $1 \leq i \leq n$, we let $u_{i}(t)$ represent the proportion of the population infected with type $i$ disease at time $t, f_{i}\left(t, u_{1}(t), u_{2}(t), \ldots, u_{n}(t)\right)$ denotes the proportion of the population newly infected with type $i$ disease per unit time, $g_{i}(t, s)$ is a certain per

\footnotetext{
'Department of Mathematical Sciences, Florida Institute of Technology, Melbourne, Florida 32901-6975, USA; e-mail: agarwal@fit.edu.

${ }^{2}$ Department of Mathematics, National University of Ireland, Galway, Ireland.

${ }^{3}$ School of Electrical and Electronic Engineering, Nanyang Technological University, 50 Nanyang

Avenue. Singapore 639798, Singapore; e-mail: ejywong@ntu.edu.sg.

(C) Australian Mathematical Society 2006. Serial-fee code 1446-1811/06
} 
unit time environmental factor associated with type $i$ disease at time $t$, and $\tau \in \mathbb{R}^{+}$is the length of time an individual remains infectious with type $i$ disease.

Throughout we shall denote $u=\left(u_{1}, u_{2}, \ldots, u_{n}\right)$. We say that $u$ is a solution of constant sign of (M1) if for each $1 \leq i \leq n$, we have $\theta_{i} u_{i}(t) \geq 0$ for $t \in \mathbb{R}$, where $\theta_{i} \in\{1,-1\}$ is fixed. Let $0<\omega<\infty$. A solution $u$ of (M1) is said to be $\omega$-periodic if $u_{i}$ is $\omega$-periodic for each $1 \leq i \leq n$. More precisely, we mean $u \in\left(A_{\omega}(\mathbb{R})\right)^{n}=A_{\omega}(\mathbb{R}) \times A_{\omega}(\mathbb{R}) \times \cdots \times A_{\omega}(\mathbb{R})(n$ times $)$ where

$$
A_{\omega}(\mathbb{R})=\{y \in B C(\mathbb{R}) \mid y(t)=y(t+\omega) \text { for all } t \in \mathbb{R}\}
$$

and $B C(\mathbb{R})$ is the space of bounded and continuous functions on $\mathbb{B}$ with values in $\mathbb{R}$.

A simplified model of (M1) when $n=1$ and $g_{i}(t, s)=1$, namely

$$
y(t)=\int_{1-\tau}^{t} f(s, y(s)) d s, \quad t \in[\tau, \infty)
$$

was first introduced in 1976 by Cooke and Kaplan [9] and Smith [19]. Using Krasnosel'skii's fixed point theorem, it is shown in [9] that (M2) has a nontrivial periodic solution provided the effective contact rate $a(t)=\lim _{y \rightarrow 0} f(t, y) / y$ exceeds a certain threshold level. On the other hand, Nussbaum [17] and Smith [19] have verified the existence of a nontrivial periodic solution when $\tau$ exceeds some threshold value. In the eighties, Leggett and Williams $[15,20]$ employed their own fixed point theorems to obtain existence criteria for (M2). Their criteria complement the threshold-type results in $[9,17,19]$.

In the present work, we have generalised the well-known (M2) to the system (M1) which not only models the spread of interdependent epidemics but also incorporates environmental factors in the modelling, and therefore is more robust for real-world applications. Further, by employing a variety of fixed point theorems we shall establish new existence results for constant-sign periodic solutions, which, when reduced to a special case, improve and generalise those in $[9,12,15]$. As a side remark, much work has been carried out on the related system of the form

$$
u_{i}(t)=\int_{0}^{1} g_{i}(t, s) f_{i}\left(s, u_{1}(s), u_{2}(s), \ldots, u_{n}(s)\right) d s, \quad t \in[0,1], 1 \leq i \leq n .
$$

The reader may refer to [2-7] which are motivated by the vast amount of literature on the existence of positive solutions $[1,8,10,11,16,18]$.

The paper is organised as follows. In Section 2, we state some well-known results in the literature which will be used to obtain the main theorems. Existence results for (M1) are developed in Section 3. We also include examples to illustrate the importance of the results obtained. 


\section{Preliminaries}

We require the definition of a Carathéodory function.

DEFINITION 2.1. A function $f: I \times \mathbb{R}^{n} \rightarrow \mathbb{R}$ is a Carathéodory function if the following conditions hold:

(a) the map $t \mapsto f(t, u)$ is measurable for all $u \in \mathbb{R}^{n}$;

(b) the map $u \mapsto f(t, u)$ is continuous for almost all $t \in I$.

The next three theorems are fixed point theorems; they are the main tools used in later sections. Theorem 2.2 is usually called Krasnosel'skii's fixed point theorem in a cone, Theorem 2.3 is known as the Leray-Schauder alternative, and Theorem 2.4 is from Leggett and Williams [14].

THEOREM 2.2 ([13]). Let $B=(B,\|\cdot\|)$ be a Banach space, and let $C \subset B$ be a cone in $B$. Assume $\Omega_{1}, \Omega_{2}$ are open bounded subsets of $B$ with $0 \in \Omega_{1}, \bar{\Omega}_{1} \subset \Omega_{2}$, and let $S: C \cap\left(\bar{\Omega}_{2} \backslash \Omega_{1}\right) \rightarrow C$ be a continuous and completely continuous operator such that either

(a) $\|S u\| \leq\|u\|, u \in C \cap \partial \Omega_{1}$, and $\|S u\| \geq\|u\|, u \in C \cap \partial \Omega_{2}$, or

(b) $\|S u\| \geq\|u\|, u \in C \cap \partial \Omega_{1}$, and $\|S u\| \leq\|u\|, u \in C \cap \partial \Omega_{2}$.

Then $S$ has a fixed point in $C \cap\left(\bar{\Omega}_{2} \backslash \Omega_{1}\right)$.

THEOREM 2.3 ([18]). Let $B$ be a Banach space with $E \subseteq B$ closed and convex. Assume $U$ is a relatively open subset of $E$ with $0 \in U$ and $S: \bar{U} \rightarrow E$ is a continuous and compact map. Then either

(a) S has a fuxed point in $\bar{U}$, or

(b) there exists $u \in \partial U$ and $\lambda \in(0,1)$ such that $u=\lambda S u$.

THEOREM 2.4 ([14]). Let $B=(B,\|\cdot\|)$ be a Banach space, $C \subset B$ a cone in $B, r_{1}>0, r_{2}>0, r_{1} \neq r_{2}$ with $R=\max \left\{r_{1}, r_{2}\right\}$ and $r=\min \left\{r_{1}, r_{2}\right\}$. Define $\bar{C}_{\eta}=\{u \in C \mid\|u\| \leq \eta\}$ and

$$
\begin{aligned}
C\left(u_{0}\right) & =\left\{u \in C \mid \text { there exists } \lambda>0 \text { such that } u \geq \lambda u_{0}\right\} \\
& =\left\{u \in C \mid \text { there exists } \lambda>0 \text { such that } u-\lambda u_{0} \in C\right\} .
\end{aligned}
$$

Let $S: \bar{C}_{R} \rightarrow C$ be a continuous and compact map such that

(a) there exists $u_{0} \in C \backslash\{0\}$ with $S u \notin u$ (equivalently $u-S u \notin C$ ) for $u \in$ $\partial \bar{C}_{r_{2}} \cap C\left(u_{0}\right)$, and

(b) $\|S u\| \leq\|u\|$ for $u \in \partial \bar{C}_{r_{1}}$.

Then $S$ has at least one fixed point $u \in C$ with $r \leq\|u\| \leq R$. 


\section{Existence Results for (M1)}

Criteria for the existence of one or more constant-sign periodic solutions of model (M1) are presented in this section. These results will be developed via various fixed point theorems.

To begin, let $0<\omega<\infty$ and let the Banach space $B=\left(A_{\omega}(\mathbb{R})\right)^{n}$ be equipped with the norm $|u|_{\omega}=\max _{1 \leq i \leq n} \sup _{t \in[0, \omega]}\left|u_{i}(t)\right|=\max _{1 \leq i \leq n}\left|u_{i}\right|_{\omega}$, where we let $\left|u_{i}\right|_{\omega}=$ $\sup _{t \in[0 . \omega]}\left|u_{i}(t)\right|, 1 \leq i \leq n$.

Define the operator $S: B \rightarrow(C(\mathbb{B}))^{n}$ by

$$
S u(t)=\left(S_{1} u(t), S_{2} u(t), \ldots, S_{n} u(t)\right), \quad t \in \mathbb{R},
$$

where

$$
S_{i} u(t)=\int_{t-\tau}^{t} g_{i}(t, s) f_{i}(s, u(s)) d s, \quad t \in \mathbb{R}, 1 \leq i \leq n .
$$

Clearly, a fixed point of the operator $S$ is a solution of the system (M1).

With $\theta_{i} \in\{1,-1\}, 1 \leq i \leq n$ fixed, define

$$
\begin{aligned}
{[0, \infty)_{i} } & = \begin{cases}{[0, \infty),} & \theta_{i}=1, \\
(-\infty, 0], & \theta_{i}=-1,\end{cases} \\
\tilde{K} & =\left\{u \in B \mid \theta_{i} u_{i}(t) \geq 0, t \in \mathbb{R}, 1 \leq i \leq n\right\}
\end{aligned}
$$

and

$$
\begin{aligned}
K & =\left\{u \in \tilde{K} \mid \theta_{j} u_{j}(t)>0 \text { for some } j \in\{1, \ldots, n\} \text { and some } t \in \mathbb{R}\right\} \\
& =\tilde{K} \backslash\{0\}
\end{aligned}
$$

Our first result uses Krasnosel'skii's fixed point theorem (Theorem 2.2).

THEOREM 3.1. Let $1 \leq p \leq \infty, q$ be such that $1 / p+1 / q=1,0<\omega<\infty$ and let $\theta_{i} \in\{1,-1\}, 1 \leq i \leq n$ be fixed. Assume the following hold for each $1 \leq i \leq n$ :

$$
\begin{aligned}
& g_{i}^{\prime}(s) \equiv g_{i}(t, s) \in L^{q}[0, \omega] \text { for each } t \in[0, \omega] \\
& \text { the map } t \mapsto g_{i}^{\prime} \text { is continuous from }[0, \omega] \text { to } L^{q}[0, \omega] \\
& g_{i}(t, s) \geq 0 \text { for all } t \in[0, \omega] \text { and a.e. } s \in[0, \omega] \\
& g_{i}(t, s+\omega)=g_{i}(t, s) \text { for all } t \in \mathbb{R} \text { and a.e. } s \in \mathbb{R} \\
& g_{i}(t+\omega, s)=g_{i}(t, s) \text { for all } t \in \mathbb{R} \text { and a.e. } s \in \mathbb{R} \\
& f_{i}:[0, \omega] \times \mathbb{B}^{n} \rightarrow \mathbb{R} \text { is a Carathéodory function; } \\
& \theta_{i} f_{i}(t, u) \geq 0 \text { for a.e. } t \in[0, \omega] \text { and all } u \in \tilde{K} ; \\
& f_{i}(t+\omega, u)=f_{i}(t, u) \text { for a.e. } t \in \mathbb{R} \text { and all } u \in \tilde{K}
\end{aligned}
$$


$\left\{\begin{array}{l}\text { for each } r>0, \text { there exists } \mu_{r}^{i} \in L^{p}[0, \omega] \text { such that }\left|u_{j}\right| \leq r, \\ 1 \leq j \leq n \text {, implies }\left|f_{i}\left(t, u_{1}, u_{2}, \ldots, u_{n}\right)\right| \leq \mu_{r}^{i}(t) \text { for a.e. } \\ t \in[0, \omega]\end{array}\right.$

$\left\{\begin{array}{l}\text { there exists a function } \psi_{i}: \prod_{j=1}^{n}[0, \infty)_{j} \rightarrow[0, \infty) \text { continuous and } \\ \text { 'nondecreasing' in the sense that for each } 1 \leq j \leq n \text {, if }\left|u_{j}\right| \leq\left|v_{j}\right|, \\ \text { then } \psi_{i}\left(u_{1}, \ldots, u_{j-1}, u_{j}, u_{j+1}, \ldots, u_{n}\right) \leq \psi_{i}\left(u_{1}, \ldots, u_{j-1}, v_{j}, u_{j+1},\right. \\ \left.\ldots, u_{n}\right), \text { a constant } \rho_{i}, 0<\rho_{i} \leq 1, \text { and a function } b_{i}: \mathbb{R} \rightarrow[0, \infty), \\ b_{i} \in L^{p}[0, \omega], b_{i}(t+\omega)=b_{i}(t) \text { for a.e. } t \in \mathbb{R}, \text { with } \rho_{i} b_{i}(t) \psi_{i}(u) \leq \\ \theta_{i} f_{i}(t, u) \leq b_{i}(t) \psi_{i}(u) \text { for a.e. } t \in[0, \omega] \text { and all } u \in \tilde{K} ;\end{array}\right.$

Ihere exists a continuous function $\phi_{i}:(0,1) \rightarrow(0, \infty)$ such that for any $0<m<1$ and $u \in[0, \infty)^{n}$, we have $\psi_{i}(m u) \geq \phi_{i}(m) \psi_{i}(u)$

$$
K_{2, i}=\inf _{t \in[0, \omega]} \int_{t-\tau}^{t} g_{i}(t, s) b_{i}(s) d s>0
$$

$\left\{\begin{array}{l}\text { there exists } 0<M_{i}<1 \text { with } M_{i} \leq \rho_{i}\left(K_{2, i} / K_{1, i}\right) \phi_{i}\left(M_{0}\right) \text {, where } \\ K_{1, i}=\sup _{t \in[0, \omega]} \int_{t-\tau}^{t} g_{i}(t, s) b_{i}(s) d s \text { and } M_{0}=\min _{1 \leq j \leq n} M_{j} \in(0,1)\end{array}\right.$

\section{Moreover, suppose}

$$
\text { there exists } \alpha>0 \text { with } \max _{1 \leq j \leq n} \psi_{j}(\alpha, \alpha, \ldots, \alpha) K_{1, j}<\alpha
$$

and

$$
\left\{\begin{array}{l}
\text { there exists } \beta>0, \beta \neq \alpha, \text { so that for each } z \in\{1,2, \ldots, n\} \text {, there } \\
\text { exists } j_{z} \in\{1,2, \ldots, n\} \text { such that for any } u \in[0, \infty)^{n} \text {, we have } \\
\psi_{j_{2}}(u) \geq a_{j_{k}}\left(u_{k}\right) \text { for each } 1 \leq k \leq n \text {, where } a_{j_{k} k}:[0, \infty) \rightarrow[0, \infty) \\
\text { is continuous, } a_{j_{k}}(x)>0 \text { if } x>0 \text {, and } \beta<\rho_{j_{2}} a_{j_{z}}\left(M_{0} \beta\right) K_{2, j} .
\end{array}\right.
$$

Then (M1) has at least one constant-sign solution $u \in\left(A_{\omega}(\mathbb{R})\right)^{n}$ satisfying

(a) $0<\alpha<|u|_{\omega}<\beta$ and $\theta_{i} u_{i}(t)>M_{i} \alpha$ for all $t \in \mathbb{R}$ and some $i \in\{1, \ldots, n\}$, if $\alpha<\beta$;

(b) $0<\beta<|u|_{\omega}<\alpha$ and $\theta_{i} u_{i}(t)>M_{i} \beta$ for all $t \in \mathbb{R}$ and some $i \in\{1, \ldots, n\}$, if $\beta<\alpha$.

ProOf. We shall employ Theorem 2.2. Define a cone $C$ in $B$ as

$$
\begin{aligned}
C & =\left\{u \in B \mid \theta_{i} u_{i}(t) \geq 0 \text { and } \theta_{i} u_{i}(t) \geq M_{i}\left|u_{i}\right|_{\omega} \text { for } 1 \leq i \leq n, t \in[0, \omega]\right\} \\
& =\left\{u \in B \mid \theta_{i} u_{i}(t) \geq 0 \text { and } \theta_{i} u_{i}(t) \geq M_{i}\left|u_{i}\right|_{\omega} \text { for } 1 \leq i \leq n, t \in \mathbb{R}\right\}
\end{aligned}
$$

where $M_{i}$ is defined in (3.15). Note that $C \subseteq \tilde{K}$. 
First, we shall show that $S$ maps $C$ into $\left(A_{\omega}(\mathbb{R})\right)^{n}$, that is, that

$$
S_{i}: C \rightarrow A_{\omega}(\mathbb{R}), \quad 1 \leq i \leq n .
$$

Let $u \in C$. Then, using (3.6), (3.7) and (3.10), we get for $t \in \mathbb{R}$ and $1 \leq i \leq n$,

$$
\begin{aligned}
S_{i} u(t+\omega) & =\int_{t+\omega-\tau}^{t+\omega} g_{i}(t+\omega, s) f_{i}(s, u(s)) d s \\
& =\int_{t-\tau}^{t} g_{i}(t+\omega, x+\omega) f_{i}(x+\omega, u(x+\omega)) d x \\
& =\int_{t-\tau}^{t} g_{i}(t+\omega, x+\omega) f_{i}(x+\omega, u(x)) d x \\
& =\int_{t-\tau}^{t} g_{i}(t, x) f_{i}(x, u(x)) d x=S_{i} u(t) .
\end{aligned}
$$

We shall next show that $S_{i} u \in C[0, \omega], 1 \leq i \leq n$. Since $u \in B \subset(B C(\mathbb{R}))^{n}$, there exists $r>0$ with $|u|_{\omega} \leq r$. Therefore (3.11) guarantees, for each $1 \leq i \leq n$, the existence of $\mu_{r}^{i} \in L^{p}[0, \omega]$, such that $\left|f_{i}(s, u(s))\right| \leq \mu_{r}^{i}(s)$, a.e. $s \in[0, \omega]$. This, together with (3.10), implies that there exists $\eta_{r}^{i} \in L^{p}[-\tau, \omega], 1 \leq i \leq n$, with

$$
\left|f_{i}(s, u(s))\right| \leq \eta_{r}^{i}(s), \quad \text { a.e. } s \in[-\tau, \omega] .
$$

(Alternatively we could assume without loss of generality that $\mu_{r}^{i}(t+\omega)=\mu_{r}^{i}(t)$ for a.e. $t \in \mathbb{R}$ and take $\eta_{r}^{i}=\mu_{r}^{i}$.) Now, for $t_{1}, t_{2} \in[0, \omega]$ with $t_{2}>t_{1}$ and $t_{2}-\tau<t_{1}$, we have for each $\mathrm{I} \leq i \leq n$.

$$
\begin{aligned}
S_{i} u\left(t_{1}\right) & -S_{i} u\left(t_{2}\right) \\
= & \int_{t_{1}-\tau}^{t_{1}} g_{i}\left(t_{1}, s\right) f_{i}(s, u(s)) d s-\int_{t_{2}-\tau}^{t_{2}} g_{i}\left(t_{2}, s\right) f_{i}(s, u(s)) d s \\
= & \int_{t_{1}-\tau}^{t_{2}-\tau} g_{i}\left(t_{1}, s\right) f_{i}(s, u(s)) d s+\int_{t_{2}-\tau}^{t_{1}}\left[g_{i}\left(t_{1}, s\right)-g_{i}\left(t_{2}, s\right)\right] f_{i}(s, u(s)) d s \\
& -\int_{t_{1}}^{t_{2}} g_{i}\left(t_{2}, s\right) f_{i}(s, u(s)) d s
\end{aligned}
$$

and so

$$
\begin{aligned}
\mid S_{i} u\left(t_{1}\right) & -S_{i} u\left(t_{2}\right) \mid \\
\leq & \left(\int_{t_{1}-\tau}^{t_{i}-\tau}\left|g_{i}^{\prime \prime}(s)\right|^{\prime \prime} d s\right)^{1 / q}\left(\int_{t_{1}-\tau}^{t_{2}-\tau}\left|f_{i}(s, u(s))\right|^{p} d s\right)^{1 / p} \\
& +\left(\int_{t_{2}-\tau}^{t_{1}}\left|g_{i}^{t_{1}}(s)-g_{i}^{t_{2}}(s)\right|^{q^{\prime}} d s\right)^{1 / t}\left(\int_{t_{2}-\tau}^{t_{1}}\left|f_{i}(s, u(s))\right|^{p} d s\right)^{1 / p}
\end{aligned}
$$


[7] On constant-sign periodic solutions in modelling the spread of interdependent epidemics

$$
+\left(\int_{t_{1}}^{t_{2}}\left|g_{i}^{i_{2}}(s)\right|^{q} d s\right)^{1 / q}\left(\int_{t_{1}}^{t_{2}}\left|f_{i}(s, u(s))\right|^{p} d s\right)^{1 / p}
$$

Using (3.21), it then follows that

$$
\begin{aligned}
\mid S_{i} u\left(t_{1}\right) & -S_{i} u\left(t_{2}\right) \mid \\
\leq & \sup _{t \in[0, \omega]}\left(\int_{-\tau}^{w-\tau}\left|g_{i}^{t}(s)\right|^{q} d s\right)^{1 / q}\left(\int_{t_{1}-\tau}^{t_{2}-\tau}\left[\eta_{r}^{i}(s)\right]^{p} d s\right)^{1 / p} \\
& +\left(\int_{-\tau}^{w}\left|g_{i}^{t_{1}}(s)-g_{i}^{t_{2}}(s)\right|^{q} d s\right)^{1 / q}\left(\int_{-\tau}^{w}\left[\eta_{r}^{i}(s)\right]^{p} d s\right)^{1 / p} \\
& +\sup _{i \in[0, \omega]}\left(\int_{0}^{w}\left|g_{i}^{t}(s)\right|^{q} d s\right)^{1 / q}\left(\int_{t_{1}}^{t_{2}}\left[\mu_{r}^{i}(s)\right]^{p} d s\right)^{1 / p} .
\end{aligned}
$$

We also note that (3.3) and (3.6) guarantee that

$$
g_{i}^{t} \in L^{q}[-\tau, \omega] \text { for } t \in[0, \omega]
$$

In view of (3.4) and (3.23), it is clear from (3.22) that $\left|S_{i} u\left(t_{1}\right)-S_{i} u\left(t_{2}\right)\right| \rightarrow 0$ as $t_{1} \rightarrow t_{2}$. Hence

$$
S_{i} u \in C[0, \omega], \quad 1 \leq i \leq n
$$

Further, noting (3.23) and (3.21), we have for $t \in[0, \omega]$ and $1 \leq i \leq n$,

$$
\left|S_{i} u(t)\right| \leq \int_{t-\tau}^{t}\left|g_{i}(t, s)\right| \eta_{r}^{i}(s) d s \leq\left(\int_{-\tau}^{\omega}\left|g_{i}^{\prime}(s)\right|^{q} d s\right)^{1 / q}\left(\int_{-\tau}^{\omega}\left[\eta_{r}^{i}(s)\right]^{p} d s\right)^{1 / p}<\infty
$$

Thus

$$
S_{i} u \text { is bounded, } 1 \leq i \leq n
$$

Having established (3.20), (3.24) and (3.25), we have completed the proof of (3.19).

Next, we shall verify that

$$
S: C \rightarrow\left(A_{\omega}(\mathbb{R})\right)^{n} \text { is continuous }
$$

Let $\left\{u^{m}\right\}, u \in C\left(\left\{u^{m}\right\}\right.$ is a sequence) with $u^{m} \rightarrow u$. Since $u^{m}, u \in B \subset(B C(\mathbb{R}))^{n}$, there exists $r>0$ with $\left|u^{m}\right|_{\omega},|u|_{\omega} \leq r$. Therefore, as in a previous argument, (3.10) and (3.11) lead to the existence of $\eta_{r}^{i} \in L^{p}[-\tau, \omega], 1 \leq i \leq n$, such that (3.21) and (3.21) $\left.\right|_{u=u^{m}}$ hold. Hence

$$
\left|f_{i}\left(s, u^{m}(s)\right)-f_{i}(s, u(s))\right| \leq 2 \eta_{r}^{i}(s), \quad \text { a.e. } s \in[-\tau, \omega]
$$


and also from (3.8) and (3.10) we have

$$
\left|f_{i}\left(s, u^{m}(s)\right)-f_{i}(s, u(s))\right| \rightarrow 0 \quad \text { pointwise for a.e. } s \in[-\tau, \omega] .
$$

Now, for $t \in[0, \omega]$ and $1 \leq i \leq n$, we find

$$
\begin{aligned}
\mid S_{i} u^{m}(t) & -S_{i} u(t) \mid \\
\leq & \int_{t-\tau}^{t}\left|g_{i}(t, s)\left[f_{i}\left(s, u^{m}(s)\right)-f_{i}(s, u(s))\right]\right| d s \\
& \leq\left(\int_{t-\tau}^{t}\left|g_{i}^{t}(s)\right|^{g} d s\right)^{1 / q}\left(\int_{t-\tau}^{t}\left|f_{i}\left(s, u^{m}(s)\right)-f_{i}(s, u(s))\right|^{p} d s\right)^{1 / p} \\
& \leq \sup _{\substack{x \in|0, \omega| \\
m \rightarrow \infty}}\left(\int_{-\tau}^{\omega}\left|g_{i}^{x}(s)\right|^{q} d s\right)^{1 / q}\left(\int_{-\tau}^{\omega}\left|f_{i}\left(s, u^{m}(s)\right)-f_{i}(s, u(s))\right|^{p} d s\right)^{1 / p}
\end{aligned}
$$

where we have used (3.23), (3.27) and (3.28). It follows that

$$
\left|S u^{m}-S u\right|_{\omega}=\max _{1 \leq i \leq n}\left|S_{i} u^{m}-S_{i} u\right|_{\omega}=\max _{i \leq i \leq n} \sup _{t \in[0, \omega]}\left|S_{i} u^{m}(t)-S_{i} u(t)\right| \stackrel{m \rightarrow \infty}{\longrightarrow} 0 .
$$

Hence we have proved (3.26).

Next, we shall show that

$$
S: C \rightarrow\left(A_{\omega}(\mathbb{R})\right)^{n} \text { is completely continuous. }
$$

Let $\Omega$ be a bounded set in $C$, that is, there exists $r>0$ such that $|u|_{\omega} \leq r$ for all $u \in \Omega$. We shall prove that $S_{i} \Omega, 1 \leq i \leq n$, is relatively compact in $A_{\omega}(\mathbb{R})$. Let $\left\{u^{m}\right\}$ be a sequence in $\Omega$. Then, for each $1 \leq i \leq n,\left\{S_{i} u^{m}\right\}$ is a sequence in $S_{i} \Omega$. Now, using once again a previous argument, (3.10) and (3.11) lead to the existence of $\eta_{r}^{i} \in L^{p}[-\tau, \omega], 1 \leq i \leq n$, such that $\left.(3.21)\right|_{u^{\prime} u^{m}}$ holds. Hence, we get for $t \in[0, \omega]$ and $1 \leq i \leq n$,

$$
\begin{aligned}
\left|S_{i} u^{m}(t)\right| & \leq\left(\int_{t-\tau}^{t}\left|g_{i}^{\prime}(s)\right|^{q} d s\right)^{1 / q}\left(\int_{t-\tau}^{t}\left|f_{i}\left(s, u^{m}(s)\right)\right|^{p} d s\right)^{1 / p} \\
& \leq \sup _{x \in|0, \omega|}\left(\int_{-\tau}^{\omega}\left|g_{i}^{x}(s)\right|^{q} d s\right)^{1 / q}\left(\int_{-\tau}^{\omega}\left[\eta_{r}^{i}(s)\right]^{p} d s\right)^{1 / p}<\infty,
\end{aligned}
$$

where we have also used (3.23). Thus $\left\{S_{i} u^{m}\right\}$ is a uniformly bounded sequence in $C[0, \omega]$. An argument similar to that in (3.22) guarantees that $\left\{S_{i} u^{m}\right\}$ is equicontinuous on $[0, \omega]$. The Arzela-Ascoli theorem guarantees an $S_{i} u \in C[0, \omega]$ and a subsequence $\left\{S_{i} u^{m_{i}}\right\}$ of $\left\{S_{i} u^{m}\right\}$ which converges uniformly on $[0, \omega]$ to $S_{i} u$. Since $S_{i} u^{m_{i}}(t+\omega)=$ 
$S_{i} u^{m_{k}}(t)$, by letting $k \rightarrow \infty$ we have $S_{i} u(t+\omega)=S_{i} u(t)$. Thus $S_{i} u \in A_{\omega}(\mathbb{R})$ and $S_{i} u^{m_{k}} \rightarrow S_{i} u$ in $A_{\omega}(\mathbb{R})$. So $S_{i}: C \rightarrow A_{\omega}(\mathbb{R})$ is completely continuous, $1 \leq i \leq n$. This completes the proof of (3.29).

We also need to show that

$$
S: C \rightarrow C .
$$

Let $u \in C$. We note that for a fixed $t \in[0, \omega]$, any $s \in[t-\tau, t]$ can be written as $s+z \omega=s_{\omega}$, where $z$ is some integer and $s_{\omega} \in[0, \omega]$. Therefore, in view of (3.5), (3.6), (3.9), (3.10) and (3.12), we get for $t \in[0, \omega]$, a.e. $s \in[t-\tau, t]$ and $1 \leq i \leq n$,

$$
\begin{aligned}
g_{i}(t, s) & =g_{i}\left(t, s_{\omega}\right) \geq 0, \\
\theta_{i} f_{i}(s, u(s)) & =\theta_{i} f_{i}\left(s_{\omega}, u(s)\right) \geq 0, \\
\theta_{i} f_{i}(s, u(s)) & =\theta_{i} f_{i}\left(s_{w}, u(s)\right) \leq b_{i}\left(s_{\omega}\right) \psi_{i}(u(s))=b_{i}(s) \psi_{i}(u(s))
\end{aligned}
$$

and

$$
\theta_{i} f_{i}(s, u(s))=\theta_{i} f_{i}\left(s_{\omega}, u(s)\right) \geq \rho_{i} b_{i}\left(s_{\omega}\right) \psi_{i}(u(s))=\rho_{i} b_{i}(s) \psi_{i}(u(s)),
$$

where we have also used the fact that $b_{i}(x+\omega)=b_{i}(x)$ for a.e. $x \in \mathbb{R}$. Then, noting (3.31) and (3.32), it is clear that

$$
\theta_{i} S_{i} u(t)=\int_{t-\tau}^{t} g_{i}(t, s) \theta_{i} f_{i}(s, u(s)) d s \geq 0, \quad t \in[0, \omega], \quad 1 \leq i \leq n .
$$

Now, using (3.12), (3.31), (3.33) and (3.35), we find for $t \in[0, \omega]$ and $1 \leq i \leq n$,

$$
\begin{aligned}
\left|S_{i} u(t)\right|=\theta_{i} S_{i} u(t) & \leq \int_{t-\tau}^{t} g_{i}(t, s) b_{i}(s) \psi_{i}(u(s)) d s \\
& \leq \psi_{i}\left(\left|u_{1}\right|_{\omega},\left|u_{2}\right|_{\omega}, \ldots,\left|u_{n}\right|_{\omega}\right) \int_{t-\tau}^{t} g_{i}(t, s) b_{i}(s) d s \\
& \leq \psi_{i}\left(\left|u_{1}\right|_{\omega},\left|u_{2}\right|_{\omega}, \ldots,\left|u_{n}\right|_{\omega}\right) K_{1, i} .
\end{aligned}
$$

This yields

$$
\left|S_{i} u\right|_{\omega} \leq K_{1 . i} \psi_{i}\left(\left|u_{1}\right|_{\omega},\left|u_{2}\right|_{\omega}, \ldots,\left|u_{n}\right|_{\omega}\right), \quad 1 \leq i \leq n .
$$

On the other hand, since $u \in C$, we have

$$
\left|u_{i}(t)\right|=\theta_{i} u_{i}(t) \geq M_{i}\left|u_{i}\right|_{\omega} \geq M_{0}\left|u_{i}\right|_{\omega}, \quad t \in \mathbb{R}, \quad 1 \leq i \leq n,
$$

where $M_{0}=\min _{1 \leq j \leq n} M_{j}$. Therefore (3.13)-(3.15), (3.31) and (3.34)-(3.37) give for $t \in[0, \omega]$ and $1 \leq i \leq n$,

$$
\theta_{i} S_{i} u(t) \geq \rho_{i} \int_{t-\tau}^{t} g_{i}(t, s) b_{i}(s) \psi_{i}(u(s)) d s
$$




$$
\begin{aligned}
& \geq \rho_{i} \psi_{i}\left(M_{0}\left|u_{1}\right|_{\omega}, M_{0}\left|u_{2}\right|_{\omega}, \ldots, M_{0}\left|u_{n}\right|_{\omega}\right) \int_{t-\tau}^{t} g_{i}(t, s) b_{i}(s) d s \\
& \geq \rho_{i} \phi_{i}\left(M_{0}\right) \psi_{i}\left(\left|u_{1}\right|_{\omega},\left|u_{2}\right|_{\omega}, \ldots,\left|u_{n}\right|_{\omega}\right) \int_{t-\tau}^{t} g_{i}(t, s) b_{i}(s) d s \\
& \geq \rho_{i} \phi_{i}\left(M_{0}\right) \psi_{i}\left(\left|u_{1}\right|_{\omega},\left|u_{2}\right|_{\omega}, \ldots,\left|u_{n}\right|_{\omega}\right) K_{2, i} \\
& \geq \rho_{i} \phi_{i}\left(M_{0}\right)\left|S_{i} u\right|_{\omega} \frac{K_{2, i}}{K_{1, i}} \geq M_{i}\left|S_{i} u\right|_{\omega} .
\end{aligned}
$$

Together with (3.35), we have shown that $S u \in C$ and hence (3.30) is proved. Let

$$
\Omega_{\alpha}=\left\{\left.u \in B|| u\right|_{\omega}<\alpha\right\} \quad \text { and } \quad \Omega_{\beta}=\left\{\left.u \in B|| u\right|_{\omega}<\beta\right\}
$$

We shall now show that

$$
|S u|_{\omega}<|u|_{\omega} \text { for } u \in C \cap \partial \Omega_{\alpha}
$$

and

$$
|S u|_{\omega}>|u|_{\omega} \quad \text { for } u \in C \cap \partial \Omega_{\beta}
$$

To verify (3.39), let $u \in C \cap \partial \Omega_{\alpha}$. Then $|u|_{\omega}=\alpha$. For $t \in[0, \omega]$, using (3.12), (3.31), (3.33) and (3.35) we find, for $1 \leq i \leq n$,

$$
\left|S_{i} u(t)\right| \leq \psi_{i}\left(|u|_{\omega},|u|_{\omega}, \ldots,|u|_{\omega}\right) \int_{t-\tau}^{t} g_{i}(t, s) b_{i}(s) d s \leq \psi_{i}(\alpha, \alpha, \ldots, \alpha) K_{1, i},
$$

which, together with (3.16), leads to

$$
|S u|_{\omega}=\max _{1 \leq i \leq n}\left|S_{i} u\right|_{\omega} \leq \max _{1 \leq i \leq n} \psi_{i}(\alpha, \alpha, \ldots, \alpha) K_{1, i}<\alpha=|u|_{\omega}
$$

Thus (3.39) is proved.

Next, let $u \in C \cap \partial \Omega_{\beta}$. Then $|u|_{\omega}=\beta=\left|u_{z}\right|_{\omega}$ for some $z \in\{1,2, \ldots, n\}$. For $t \in[0, \omega]$, using (3.17), (3.31), (3.34), (3.35) and (3.37), we get for some $j_{z} \in\{1,2, \ldots, n\}$,

$$
\begin{aligned}
\left|S_{j_{:}} u(t)\right| & \geq \rho_{j:} \int_{t-\tau}^{t} g_{j:}(t, s) b_{j:}(s) \psi_{j:}(u(s)) d s \\
& \geq \rho_{j_{i}} \psi_{j:}\left(M_{0}\left|u_{1}\right|_{\omega}, M_{0}\left|u_{2}\right|_{\omega}, \ldots, M_{0}\left|u_{n}\right|_{\omega}\right) \int_{t-\tau}^{t} g_{j:}(t, s) b_{j:}(s) d s \\
& \geq \rho_{j:} a_{j:}\left(M_{0}\left|u_{z}\right|_{\omega}\right) K_{2, j:}=\rho_{j:} a_{j_{z}:}\left(M_{0} \beta\right) K_{2, j:}>\beta=|u|_{\omega} .
\end{aligned}
$$

Thus

$$
|S u|_{\omega} \geq\left|S_{j:} u\right|_{\omega}>|u|_{\omega}
$$


and (3.40) is true.

Now Theorem 2.2 guarantees that $S$ has a fixed point $u$ with $u \in C \cap\left(\bar{\Omega}_{\alpha} \backslash \Omega_{\beta}\right)$ if $\beta<\alpha$, whereas $u \in C \cap\left(\bar{\Omega}_{\beta} \backslash \Omega_{\alpha}\right)$ if $\alpha<\beta$. Hence, equivalently (MI) has a constant-sign solution $u \in\left(A_{\omega}(\mathbb{R})\right)^{n}$ with $\min \{\alpha, \beta\} \leq|u|_{\omega} \leq \max \{\alpha, \beta\}$. Note that $|u|_{\omega} \neq \alpha$ and $|u|_{\omega} \neq \beta$. To see this, suppose $|u|_{\omega}=\alpha$ or $|u|_{\omega}=\beta$. Then, since $u=S u$ we have, noting (3.41) and (3.42),

$$
\alpha=|u|_{\omega}=|S u|_{\omega} \leq \max _{1 \leq i \leq n} \psi_{i}(\alpha, \alpha, \ldots, \alpha) K_{1, i}<\alpha=|u|_{\omega}
$$

or $\beta=|u|_{\omega}=|S u|_{\omega} \geq\left|S_{j_{z}} u\right|_{\omega}>|u|_{\omega}=\beta$, which are contradictions. Hence

$$
\min \{\alpha, \beta\}<|u|_{\omega}<\max \{\alpha, \beta\}
$$

Finally, $|u|_{\omega}=\left|u_{i}\right|_{\omega}$ for some $i \in\{1,2, \ldots, n\}$. Since $u \in C$, we have, for $t \in \mathbb{R}$, $\theta_{i} u_{i}(t) \geq M_{i}\left|u_{i}\right|_{\omega}=M_{i}|u|_{\omega}>M_{i} \min \{\alpha, \beta\}$.

REMARK 3.2. In Theorem 3.1, it is possible to replace (3.3), (3.4), (3.6)-(3.8) and (3.11) with the following:

$$
\begin{aligned}
& g_{i}^{t}(s) \equiv g_{i}(t, s) \in L^{1}[0, \omega] \text { for each } t \in[0, \omega] \\
& \text { the map } t \mapsto g_{i}^{\prime} \text { is continuous from }[0, \omega] \text { to } L^{\prime}[0, \omega] \\
& \begin{cases}g_{i}(t, s) \geq 0 & \text { for all } t \in[0, \omega] \text { and a.e. } s \in[t-\tau, t], \\
g_{i}(t+\omega, s+\omega)=g_{i}(t, s) & \text { for all } t \in \mathbb{R} \text { and a.e. } s \in \mathbb{R} ;\end{cases} \\
& f_{i}:[0, \omega] \times \mathbb{B}^{n} \rightarrow \mathbb{R} \text { is continuous. }
\end{aligned}
$$

As in [7, Section 3], we need only notice that (3.43)-(3.46) imply that $S: C \rightarrow C$ is continuous and completely continuous.

Our next result gives the existence of multiple constant-sign periodic solutions.

THEOREM 3.3. Let $1 \leq p \leq \infty, q$ be such that $1 / p+1 / q=1,0<\omega<\infty$ and let $\theta_{i} \in\{1,-1\}, 1 \leq i \leq n$, be fixed. Assume that (3.3)-(3.15) hold for each $1 \leq i \leq n$. Let (3.16) be satisfied for $\alpha=\alpha_{\ell}, \ell=1, \ldots, k$, and (3.17) be satisfied for $\beta=\beta_{\ell}$, $\ell=1, \ldots, m$.

(a) If $m=k+1$ and $0<\beta_{1}<\alpha_{1}<\cdots<\beta_{k}<\alpha_{k}<\beta_{k+1}$, then (M1) has (at least) $2 k$ constant-sign solutions $u^{1}, \ldots, u^{2 k} \in\left(A_{\omega}(\mathbb{R})\right)^{n}$ such that

$$
0<\beta_{1}<\left|u^{1}\right|_{\omega}<\alpha_{1}<\left|u^{2}\right|_{\omega}<\beta_{2}<\cdots<\alpha_{k}<\left|u^{2 k}\right|_{\omega}<\beta_{k+1} .
$$

(b) If $m=k$ and $0<\beta_{1}<\alpha_{1}<\cdots<\beta_{k}<\alpha_{k}$, then (M1) has (at least) $2 k-1$ constant-sign solutions $u^{\prime}, \ldots, u^{2 k-1} \in\left(A_{\omega}(\mathbb{R})\right)^{n}$ such that

$$
0<\beta_{1}<\left|u^{1}\right|_{\omega}<\alpha_{1}<\left|u^{2}\right|_{\omega}<\beta_{2}<\cdots<\beta_{k}<\left|u^{2 k-1}\right|_{\omega}<\alpha_{k} .
$$


(c) If $k=m+1$ and $0<\alpha_{1}<\beta_{1}<\cdots<\alpha_{m}<\beta_{m}<\alpha_{m+1}$, then (M1) has (at least $) 2 m$ constant - sign solutions $u^{1}, \ldots, u^{2 m} \in\left(A_{\omega}(\mathbb{R})\right)^{n}$ such that

$$
0<\alpha_{1}<\left|u^{\prime}\right|_{\omega}<\beta_{1}<\left|u^{2}\right|_{\omega}<\alpha_{2}<\cdots<\beta_{m}<\left|u^{2 m}\right|_{\omega}<\alpha_{m+1} .
$$

(d) If $k=m$ and $0<\alpha_{1}<\beta_{1}<\cdots<\alpha_{k}<\beta_{k}$, then (M1) has (at least) $2 k-1$ constant-sign solutions $u^{1}, \ldots, u^{2 k-1} \in\left(A_{\omega}(\mathbb{R})\right)^{n}$ such that

$$
0<\alpha_{1}<\left|u^{1}\right|_{\omega}<\beta_{1}<\left|u^{2}\right|_{\omega}<\alpha_{2}<\cdots<\alpha_{k}<\left|u^{2 k-1}\right|_{\omega}<\beta_{k} .
$$

EXAMPLE 1. Consider the nonlinear system of integral equations

$$
u_{i}(t)=\int_{t-\tau}^{t} g_{i}(t, s) \theta_{i} h_{i}(s)\left[\left|u_{1}(s)\right|^{\gamma}+\left|u_{2}(s)\right|^{\gamma}+\cdots+\left|u_{n}(s)\right|^{\gamma}\right] d s,
$$

$t \in \mathbb{R}, 1 \leq i \leq n$, where $0<\gamma<1$ and $\theta_{i} \in\{-1,1\}, 1 \leq i \leq n$, are fixed. For each $1 \leq i \leq n$, assume (3.3)-(3.7) hold,

$$
\left\{\begin{array}{l}
\text { there exists } \omega>0 \text { with } h_{i}(t+\omega)=h_{i}(t) \text { for a.e. } t \in \mathbb{R}, \\
h_{i} \text { is nonnegative and } h_{i} \in L^{p}[0, \omega]
\end{array}\right.
$$

and

$$
\inf _{i \in[0,(\omega)} \int_{t-\tau}^{t} g_{i}(t, s) h_{i}(s) d s>0 .
$$

Then (3.47) has at least one constant-sign solution $u \in\left(A_{\omega}(\mathbb{R})\right)^{n}$ such that

$$
\left\{\begin{array}{l}
0<\min \{\alpha, \beta\}<|u|_{\omega}<\max \{\alpha, \beta\} \text { and } \\
\theta_{k} u_{k}(t)>M_{k} \min \{\alpha, \beta\} \text { for all } t \in \mathbb{R} \text { and some } k \in\{1, \ldots, n\},
\end{array}\right.
$$

where

$$
M_{i}=\frac{K_{2 . i}}{K_{1 . i}}\left(\min _{1 \leq j \leq n} \frac{K_{2 . j}}{K_{1, j}}\right)^{\gamma /(1-\gamma)} \in(0,1), \quad 1 \leq i \leq n
$$

with

$$
K_{2 . i}=\inf _{t \in \mid 0, \omega]} \int_{t-\tau}^{t} g_{i}(t, s) h_{i}(s) d s, \quad K_{1, i}=\sup _{t \in[0, \omega]} \int_{t-\tau}^{t} g_{i}(t, s) h_{i}(s) d s
$$

and $\alpha, \beta$ are positive numbers satisfying

$$
\alpha>\left(n \max _{1 \leq j \leq n} K_{1 . j}\right)^{1 /(1-\gamma)} \text { and } \beta<M_{0}^{\gamma /(1-\gamma)}\left(\min _{1 \leq j \leq n} K_{2 . j}\right)^{1 /(1-\gamma)},
$$


where $M_{0}=\min _{1 \leq j \leq n} M_{j} \in(0,1)$.

To see that the above is true, we shall apply Theorem 3.1 with

$$
\left\{\begin{array}{l}
f_{i}(t, u)=\theta_{i} h_{i}(t)\left[\left|u_{1}\right|^{\gamma}+\left|u_{2}\right|^{\gamma}+\cdots+\left|u_{n}\right|^{\gamma}\right], \\
\rho_{i}=1, \quad b_{i}=h_{i}, \quad \psi_{i}(u)=\left|u_{1}\right|^{\gamma}+\left|u_{2}\right|^{\gamma}+\cdots+\left|u_{n}\right|^{\gamma}, \\
\phi_{i}(m)=m^{\gamma} \quad \text { and } \quad a_{j_{i} k}(x)=x^{\gamma}, \quad 1 \leq i, j_{i}, k \leq n .
\end{array}\right.
$$

Note that (3.8)-(3.14) are clearly satisfied. Next, the inequality in condition (3.15) is reduced to $M_{i} \leq\left(K_{2, i} / K_{1, i}\right) M_{0}^{\gamma}, 1 \leq i \leq n$, which will be satisfied if we set

$$
M_{i}=\frac{K_{2, i}}{K_{1, i}} M_{0}^{\gamma}, \quad 1 \leq i \leq n
$$

It follows immediately that $M_{0}=\min _{1 \leq j \leq n} M_{j}=\min _{1 \leq j \leq n}\left(K_{2, j} / K_{1, j}\right) M_{0}^{\gamma}$ or

$$
M_{0}=\left(\min _{1 \leq j \leq n} \frac{K_{2, j}}{K_{1, j}}\right)^{1 /(1-\gamma)} .
$$

Substituting (3.56) in (3.55) yields (3.51).

Further, (3.16) holds since noting (3.54) we find

$$
\max _{1 \leq j \leq n} \psi_{j}(\alpha, \alpha, \ldots, \alpha) K_{1, j}=n \alpha^{\gamma} \max _{1 \leq j \leq n} K_{1, j}<\alpha^{\gamma} \alpha^{1-\gamma}=\alpha,
$$

where we have also used (3.53).

Finally, (3.17) is fulfilled since in view of (3.53) and (3.54) we have

$$
\begin{aligned}
\rho_{j_{z}} a_{j_{z} z}\left(M_{0} \beta\right) K_{2, j:} & =\left(M_{0} \beta\right)^{\gamma} K_{2 . j_{z}} \geq\left(M_{0} \beta\right)^{\gamma} \min _{1 \leq j \leq n} K_{2, j} \\
& >\left(M_{0} \beta\right)^{\gamma} \beta^{1-\gamma} M_{0}^{-\gamma}=\beta .
\end{aligned}
$$

We now conclude from Theorem 3.1 that the system (3.47) has at least one constantsign solution $u \in\left(A_{\omega}(\mathbb{R})\right)^{n}$ satisfying (3.50)-(3.53).

The next result uses the nonlinear alternative (Theorem 2.3) to show the existence of a periodic solution (which need not be of constant sign).

THEOREM 3.4. Let $1 \leq p \leq \infty, q$ be such that $1 / p+1 / q=1$ and $0<\omega<\infty$. For each $1 \leq i \leq n$, assume that (3.3), (3.4), (3.6)-(3.8) and (3.11) hold and

$$
f_{i}(t+\omega, u)=f_{i}(t, u) \text { for a.e. } t \in \mathbb{R} \text { and } u \in \mathbb{R}^{n} .
$$

Suppose there exists a constant $c$, independent of $\lambda$, such that

$$
|u|_{\omega} \neq c
$$


for any solution $u \in\left(A_{\omega}(\mathbb{R})\right)^{n}$ of the system

$$
u_{i}(t)=\lambda \int_{t-\tau}^{t} g_{i}(t, s) f_{i}(s, u(s)) d s, \quad t \in \mathbb{R}, 1 \leq i \leq n,
$$

where $\lambda \in(0,1)$. Then, (M1) has at least one solution $u \in\left(A_{\omega}(\mathbb{R})\right)^{n}$ with $|u|_{\omega} \leq c$.

Proof. We shall apply Theorem 2.3. Let $E=B=\left(\left(A_{\omega}(\mathbb{R})\right)^{n},|\cdot|_{\omega}\right)$ and $U=\left\{\left.u \in B|| u\right|_{\omega}<c\right\}$. Clearly, a solution of $(3.59)_{\lambda}$ is a fixed point of the equation $u=\lambda S u$. As seen in the proof of Theorem 3.1, (3.3), (3.4), (3.6)-(3.8), (3.11) and (3.57) guarantee that $S: B \rightarrow B$ is continuous and completely continuous. In view of (3.58), we cannot have conclusion (b) of Theorem 2.3 , hence conclusion (a) of Theorem 2.3 must hold, that is, $S$ has a fixed point in $\bar{U}$, or equivalently the system (M1) has a solution $u \in \bar{U}$ with $|u|_{\omega} \leq c$.

Using Theorem 3.4, we shall obtain the existence of a constant-sign periodic solution in the next result.

THEOREM 3.5. Let $1 \leq p \leq \infty$, $q$ be such that $1 / p+1 / q=1,0<\omega<\infty$ and let $\theta_{i} \in\{1,-1\}, 1 \leq i \leq n$, be fixed. For each $1 \leq i \leq n$, assume that (3.3)-(3.9), (3.11) and (3.57) hold and

$$
\left\{\begin{array}{l}
\text { there exists a function } \psi_{i}: \prod_{j=1}^{n}[0, \infty)_{j} \rightarrow[0, \infty) \text { continu- } \\
\text { ous and 'nondecreasing' in the sense that for each } 1 \leq j \leq \\
n, \text { if }\left|u_{j}\right| \leq\left|v_{j}\right|, \text { then } \psi_{i}\left(u_{1}, \ldots, u_{j-1}, u_{j}, u_{j+1}, \ldots, u_{n}\right) \leq \\
\psi_{i}\left(u_{1}, \ldots, u_{j-1}, v_{j}, u_{j+1}, \ldots, u_{n}\right), \text { and a function } b_{i}: \mathbb{R} \rightarrow \\
{[0, \infty), b_{i} \in L^{p}[0, \omega], b_{i}(t+\omega)=b_{i}(t) \text { for a.e. } t \in \mathbb{R}, \text { with }} \\
\theta_{i} f_{i}(t, u) \leq b_{i}(t) \psi_{i}(u) \text { for a.e. } t \in[0, \omega] \text { and all } u \in \tilde{K} .
\end{array}\right.
$$

Moreover, suppose

$$
\left\{\begin{array}{l}
\text { there exists } \alpha>0 \text { with } \max _{1 \leq j \leq n} \psi_{j}(\alpha, \alpha, \ldots, \alpha) K_{1, j}<\alpha, \text { where } \\
K_{1, j}=\sup _{t \in|0, \omega|} \int_{t-\tau}^{t} g_{j}(t, s) b_{j}(s) d s .
\end{array}\right.
$$

Then, (M1) has at least one constant-sign solution $u \in\left(A_{\omega}(\mathbb{R})\right)^{n}$ with $|u|_{\omega}<\alpha$.

ProOF. We shall employ Theorem 3.4. To begin, we consider the system

$$
u_{i}(t)=\int_{t-\tau}^{t} g_{i}(t, s) \hat{f}_{i}(s, u(s)) d s, \quad t \in \mathbb{R}, \quad I \leq i \leq n
$$

where $\hat{f}_{i}: \mathbb{R} \times \mathbb{R}^{n} \rightarrow \mathbb{R}$ is defined by

$$
\hat{f}_{i}\left(t, u_{1}, u_{2}, \ldots, u_{n}\right)=f_{i}\left(t, \theta_{1}\left|u_{1}\right|, \theta_{2}\left|u_{2}\right|, \ldots, \theta_{n}\left|u_{n}\right|\right), \quad 1 \leq i \leq n .
$$


We shall prove that (3.62) has a solution. For this, we consider the system

$$
u_{i}(t)=\lambda \int_{t-\tau}^{t} g_{i}(t, s) \hat{f}_{i}(s, u(s)) d s, \quad t \in \mathbb{R}, 1 \leq i \leq n,
$$

where $\lambda \in(0,1)$. Let $u \in\left(A_{\omega}(\mathbb{R})\right)^{n}$ be any solution of $(3.64)_{\lambda}$. If we can show that

$$
|u|_{\omega} \neq \alpha,
$$

then by Theorem 3.4 it follows that (3.62) has a solution.

Now, using (3.5), (3.9), the fact that $\left(\theta_{1}\left|u_{1}\right|, \theta_{2}\left|u_{2}\right|, \ldots, \theta_{n}\left|u_{n}\right|\right) \in \tilde{K},(3.31),(3.32)$ and (3.63) we get for $t \in[0, \omega]$ and $1 \leq i \leq n$,

$$
\begin{aligned}
\theta_{i} u_{i}(t) & =\lambda \int_{t-\tau}^{t} g_{i}(t, s) \theta_{i} \hat{f}_{i}\left(s, u_{1}(s), u_{2}(s), \ldots, u_{n}(s)\right) d s \\
& =\lambda \int_{t-\tau}^{t} g_{i}(t, s) \theta_{i} f_{i}\left(s, \theta_{1}\left|u_{1}(s)\right|, \theta_{2}\left|u_{2}(s)\right|, \ldots, \theta_{n}\left|u_{n}(s)\right|\right) d s \geq 0,
\end{aligned}
$$

which means that

$$
\left|u_{i}(t)\right|=\theta_{i} u_{i}(t), t \in[0, \omega], 1 \leq i \leq n .
$$

An application of (3.33),(3.60) and (3.66) yields for $t \in[0, \omega]$ and $1 \leq i \leq n$,

$$
\begin{aligned}
\left|u_{i}(t)\right|=\theta_{i} u_{i}(t) & \leq \int_{t-\tau}^{t} g_{i}(t, s) \theta_{i} f_{i}\left(s, \theta_{1}\left|u_{1}(s)\right|, \ldots, \theta_{n}\left|u_{n}(s)\right|\right) d s \\
& \leq \int_{t-\tau}^{t} g_{i}(t, s) b_{i}(s) \psi_{i}\left(\theta_{1}\left|u_{1}(s)\right|, \ldots, \theta_{n}\left|u_{n}(s)\right|\right) d s \\
& \leq \psi_{i}\left(|u|_{\omega}, \ldots,|u|_{\omega}\right) \int_{t-\tau}^{t} g_{i}(t, s) b_{i}(s) d s \\
& \leq \psi_{i}\left(|u|_{\omega}, \ldots,|u|_{\omega}\right) K_{1, i} .
\end{aligned}
$$

This immediately leads to

$$
\left|u_{i}\right|_{\omega} \leq \psi_{i}\left(|u|_{\omega}, \ldots,|u|_{\omega}\right) K_{1, i}, \quad 1 \leq i \leq n .
$$

Now $|u|_{\omega}=\left|u_{z}\right|_{\omega}$ for some $z \in\{1,2, \ldots, n\}$. Then it follows from (3.67) that

$$
|u|_{\omega} \leq \psi_{z}\left(|u|_{\omega}, \ldots,|u|_{\omega}\right) K_{1.2} .
$$

Noting (3.61) and (3.68), we conclude that $|u|_{\omega} \neq \alpha$. Hence (3.65) is proved.

It now follows from Theorem 3.4 that the system (3.62) has a solution $u^{*}=$ $\left(u_{1}^{*}, u_{2}^{*}, \ldots, u_{n}^{*}\right) \in\left(A_{\omega}(\mathbb{R})\right)^{n}$ with $\left\|u^{*}\right\| \leq \alpha$, and

$$
u_{i}^{*}(t)=\int_{t-\tau}^{t} g_{i}(t, s) \hat{f}_{i}\left(s, u^{*}(s)\right) d s, \quad t \in \mathbb{R}, 1 \leq i \leq n .
$$


Using a similar argument as above, together with $u^{*} \in\left(A_{\omega}(\mathbb{B})\right)^{n}$, it can be easily seen that

$$
\left|u_{i}^{*}(t)\right|=\theta_{i} u_{i}^{*}(t), \quad t \in \mathbb{R}, 1 \leq i \leq n,
$$

and

$$
\left|u^{*}\right|_{\omega} \neq \alpha .
$$

Therefore $u^{*}$ is of constant sign and $\left|u^{*}\right|_{\omega}<\alpha$. Further, using (3.63) and (3.69), we have for $t \in \mathbb{R}$ and $1 \leq i \leq n$,

$$
\begin{aligned}
u_{i}^{*}(t) & =\int_{t-\tau}^{t} g_{i}(t, s) \hat{f}_{i}\left(s, u^{*}(s)\right) d s=\int_{t-\tau}^{t} g_{i}(t, s) f_{i}\left(s, \theta_{1}\left|u_{1}^{*}(s)\right|, \ldots, \theta_{n}\left|u_{n}^{*}(s)\right|\right) d s \\
& =\int_{t-\tau}^{t} g_{i}(t, s) f_{i}\left(s, \theta_{1}^{2} u_{1}^{*}(s), \ldots, \theta_{n}^{2} u_{n}^{*}(s)\right) d s \\
& =\int_{t-\tau}^{t} g_{i}(t, s) f_{i}\left(s, u_{1}^{*}(s), \ldots, u_{n}^{*}(s)\right) d s .
\end{aligned}
$$

Hence $u^{*}$ is in fact a solution of (M1). The proof is now complete.

In Theorem 3.5, it is possible for $|\boldsymbol{u}|_{\omega}$ to be zero. However, we can combine Theorem 3.5 with Theorem 3.1 to obtain the existence of multiple nontrivial constantsign periodic solutions, stated as the next result.

THEOREM 3.6. Let $1 \leq p \leq \infty$, $q$ be such that $1 / p+1 / q=1,0<\omega<\infty$ and let $\theta_{i} \in\{1,-1\}, 1 \leq i \leq n$, be fixed. Assume that (3.3)-(3.15) and (3.57) hold for each $1 \leq i \leq n$. Let (3.16) be satisfied for $\alpha=\alpha_{\ell}, \ell=1,2, \ldots, k$, and (3.17) be satisfied for $\beta=\beta_{\ell}, \ell=1,2, \ldots, m$.

(a) If $m=k+1$ and $0<\beta_{1}<\alpha_{1}<\cdots<\beta_{k}<\alpha_{k}<\beta_{k+1}$, then (M1) has (at least) $2 k$ constant-sign solutions $u^{1}, \ldots, u^{2 k} \in\left(A_{\omega}(\mathbb{R})\right)^{n}$ such that

$$
0<\beta_{1}<\left|u^{1}\right|_{\omega}<\alpha_{1}<\left|u^{2}\right|_{\omega}<\beta_{2}<\cdots<\alpha_{k}<\left|u^{2 k}\right|_{\omega}<\beta_{k+1} .
$$

(b) If $m=k$ and $0<\beta_{1}<\alpha_{1}<\cdots<\beta_{k}<\alpha_{k}$, then (M1) has (at least) $2 k-1$ constant-sign solutions $u^{1}, \ldots, u^{2 k-1} \in\left(A_{\omega}(\mathbb{B})\right)^{n}$ such that

$$
0<\beta_{1}<\left|u^{1}\right|_{\omega}<\alpha_{1}<\left|u^{2}\right|_{\omega}<\beta_{2}<\cdots<\beta_{k}<\left|u^{2 k-1}\right|_{\omega}<\alpha_{k} .
$$

(c) If $k=m+1$ and $0<\alpha_{1}<\beta_{1}<\cdots<\alpha_{m}<\beta_{m}<\alpha_{m+1}$, then (M1) has (at least) $2 m+1$ constant-sign solutions $u^{0}, \ldots, u^{2 m} \in\left(A_{\omega}(\mathbb{R})\right)^{n}$ such that

$$
0 \leq\left|u^{0}\right|_{\omega}<\alpha_{1}<\left|u^{1}\right|_{\omega}<\beta_{1}<\left|u^{2}\right|_{\omega}<\alpha_{2}<\cdots<\beta_{m}<\left|u^{2 m}\right|_{\omega}<\alpha_{m+1} .
$$


(d) If $k=m$ and $0<\alpha_{1}<\beta_{1}<\cdots<\alpha_{k}<\beta_{k}$, then (M1) has (at least) $2 k$ constant-sign solutions $u^{0}, \ldots, u^{2 k-1} \in\left(A_{\omega}(\mathbb{R})\right)^{n}$ such that

$$
0 \leq\left|u^{0}\right|_{\omega}<\alpha_{1}<\left|u^{1}\right|_{\omega}<\beta_{1}<\left|u^{2}\right|_{\omega}<\alpha_{2}<\cdots<\alpha_{k}<\left|u^{2 k-1}\right|_{\omega}<\beta_{k} .
$$

PROOF. In (a) and (b), we just apply Theorem 3.1 repeatedly. In (c) and (d), Theorem 3.5 is used to obtain the existence of $u^{0} \in\left(A_{\omega}(\mathbb{R})\right)^{n}$ with $0 \leq\left\|u^{0}\right\|<\alpha_{1}$; the results then follow by repeated use of Theorem 3.1.

Our next result makes use of Leggett and Williams' fixed point theorem (Theorem 2.4).

THEOREM 3.7. Let $1 \leq p \leq \infty, q$ be such that $1 / p+1 / q=1,0<\omega<\infty$ and let $\theta_{i} \in\{1,-1\}, 1 \leq i \leq n$, be fixed. Assume that (3.3)-(3.11) and (3.60) hold for each $1 \leq i \leq n$. Moreover, suppose

$\exists j \in\{1, \ldots, n\}$ such that $K_{2, j}=\inf _{t \in[0, \omega]} \int_{t-\tau}^{t} g_{j}(t, s) b_{j}(s) d s>0$;

for the same $j$ as in (3.71), there exists $r>0$ with $r<$ $\phi_{j}(r, \ldots, r) K_{2, j}$, where $\phi_{j}: \prod_{i=1}^{n}[0, \infty)_{i} \rightarrow[0, \infty)$ is continuous, $\phi_{j}\left(u_{1}, \ldots, u_{n}\right) /\left|u_{j}\right|$ is 'nonincreasing' in the sense that for each $1 \leq k \leq n$, if $0<\left|u_{k}\right| \leq\left|v_{k}\right| \leq r$, then $\phi_{j}\left(u_{1}, \ldots, u_{n}\right) /\left|u_{j}\right| \geq$ $\phi_{j}\left(v_{1}, \ldots, v_{n}\right) /\left|v_{j}\right|$, and $b_{j}(t) \phi_{j}(u) \leq \theta_{j} f_{j}(t, u)$ for a.e. $t \in[0, \omega]$ and all $u \in K$

and

$\left\{\begin{array}{l}\text { there exists } R(\neq r) \text { with } R>\max _{1 \leq i \leq n} \psi_{i}(R, \ldots, R) K_{1, i}, \text { where } \\ K_{1, i}=\sup _{t \in[0, \omega]} \int_{i-\tau}^{t} g_{i}(t, s) b_{i}(s) d s .\end{array}\right.$

Then (M1) has at least one constant-sign solution $u \in\left(A_{\omega}(\mathbb{R})\right)^{n}$ with

$$
\min \{r, R\} \leq|u|_{\omega} \leq \max \{r, R\} \text { and }|u|_{\omega} \neq R
$$

ProOF. Let $B=\left(\left(A_{\omega}(\mathbb{R})\right)^{n},|\cdot|_{\omega}\right)$ and

$$
\begin{aligned}
C & =\left\{u \in B \mid \text { for each } 1 \leq i \leq n, \theta_{i} u_{i}(t) \geq 0 \text { for } t \in[0, \omega]\right\} \\
& =\left\{u \in B \mid \text { for each } 1 \leq i \leq n, \theta_{i} u_{i}(t) \geq 0 \text { for } t \in \mathbb{R}\right\} .
\end{aligned}
$$

Also, let $u_{0} \equiv\left(\theta_{1} \ldots \theta_{n}\right)$. Then

$$
\begin{aligned}
C\left(u_{0}\right) & =\left\{u \in C \mid \text { there exists } \lambda>0 \text { with } u(t)-\lambda u_{0} \in C \text { for } t \in[0, \omega]\right\} \\
& =\left\{u \in C \mid \text { for each } 1 \leq i \leq n, \theta_{i} u_{i}(t)>0 \text { for } t \in[0, \omega]\right\} .
\end{aligned}
$$


As seen in the proof of Theorem 3.1, conditions (3.3)-(3.11) guarantee that $S: C \rightarrow C$ is continuous and completely continuous. To apply Theorem 2.4 , we shall first show that

$$
|S u|_{\omega} \leq|u|_{\omega} \text { for } u \in \partial \bar{C}_{R}
$$

Let $u \in \partial \bar{C}_{R}$. Then $|u|_{\omega}=R$. Using (3.33) and (3.60), we find for $t \in[0, \omega]$ and $1 \leq i \leq n$,

$$
\begin{aligned}
\left|S_{i} u(t)\right|=\theta_{i} S_{i} u(t) & \leq \int_{t-\tau}^{t} g_{i}(t, s) b_{i}(s) \psi_{i}(u(s)) d s \\
& \leq \psi_{i}\left(\left|u_{1}\right|_{\omega}, \ldots,\left|u_{n}\right|_{\omega}\right) \int_{t-\tau}^{t} g_{i}(t, s) b_{i}(s) d s \leq \psi_{i}(R, \ldots, R) K_{1, i} .
\end{aligned}
$$

This yields, together with (3.73),

$$
|S u|_{\omega}=\max _{1 \leq i \leq n}\left|S_{i} u\right|_{\omega} \leq \max _{1 \leq i \leq n} \psi_{i}(R, \ldots, R) K_{1, i}<R=|u|_{\omega}
$$

and hence (3.75) is proved.

Next, we shall verify that

$S u \notin u, \quad$ that is, $u-S u \notin C, \quad$ for $u \in \partial \bar{C}_{r} \cap C\left(u_{0}\right)$.

Let $u \in \partial \bar{C}_{r} \cap C\left(u_{0}\right)$. Then

$$
|u|_{\omega}=r \quad \text { and } \quad r \geq \theta_{i} u_{i}(t)>0, \quad t \in[0, \omega], 1 \leq i \leq n .
$$

By a similar argument as in getting (3.31)-(3.34) from (3.72) we have the following for $u \in C, t \in[0, \omega]$, a.e. $s \in[t-\tau, t]$ and some $j \in\{1,2, \ldots, n\}$ (the same $j$ as in (3.71)):

$$
\theta_{j} f_{j}(s, u(s))=\theta_{j} f_{j}\left(s_{\omega}, u(s)\right) \geq b_{j}\left(s_{\omega}\right) \phi_{j}(u(s))=b_{j}(s) \phi_{j}(u(s)) .
$$

Thus, for $t \in[0, \omega]$ and the same $j$ as in (3.71), using (3.78) and (3.79) we get

$$
\begin{aligned}
\theta_{j} S_{j} u(t) & \geq \int_{t-\tau}^{t} g_{j}(t, s) b_{j}(s) \phi_{j}(u(s)), d s \\
& =\int_{t-\tau}^{t} g_{j}(t, s) b_{j}(s) \frac{\phi_{j}(u(s))}{\theta_{j} u_{j}(s)} \theta_{j} u_{j}(s) d s \\
& \geq \frac{\phi_{j}(r, \ldots, r)}{r} \int_{t-\tau}^{t} g_{j}(t, s) b_{j}(s) \theta_{j} u_{j}(s) d s .
\end{aligned}
$$


Let $t_{0 . j} \in[0, \omega]$ be such that $\inf _{t \in[0, \omega]} \theta_{j} u_{j}(t)=\theta_{j} u_{j}\left(t_{0, j}\right)>0$. Then it follows from (3.72) and (3.80) that for $t \in[0, \omega]$,

$$
\begin{aligned}
\theta_{j} S_{j} u(t) & \geq \frac{\phi_{j}(r, \ldots, r)}{r} \theta_{j} u_{j}\left(t_{0, j}\right) \int_{t-\tau}^{t} g_{j}(t, s) b_{j}(s) d s \\
& \geq\left[\frac{\phi_{j}(r, \ldots, r)}{r} K_{2, j}\right] \theta_{j} u_{j}\left(t_{0, j}\right)>\theta_{j} u_{j}\left(t_{0, j}\right) .
\end{aligned}
$$

Thus in particular we have $\theta_{j} S_{j} u\left(t_{0, j}\right)>\theta_{j} u_{j}\left(t_{0, j}\right)$, and so (3.77) is proved.

It now follows from Theorem 2.4 that system (M1) has a constant-sign solution $u \in\left(A_{\omega}(\mathbb{R})\right)^{n}$ with $\min \{r, R\} \leq|u|_{\omega} \leq \max \{r, R\}$. Note that $|u|_{\omega} \neq R$. In fact, if $|u|_{\omega}=R$, then from (3.76) we have $|u|_{\omega}=|S u|_{\omega}<R=|u|_{\omega}$ which is a contradiction. This completes the proof.

REMARK 3.8. If the inequality in (3.73) is changed to

$$
R \geq \max _{1 \leq i \leq n} \psi_{i}(R, \ldots, R) K_{1, i}
$$

then (3.74) is correspondingly changed to $\min \{r, R\} \leq|u|_{\omega} \leq \max \{r, R\}$.

REMARK 3.9. Theorem 3.7 improves the results obtained in [9].

A repeated application of Theorem 3.7 yields the existence of multiple solutions as follows.

THEOREM 3.10. Let $1 \leq p \leq \infty, q$ be such that $1 / p+1 / q=1,0<\omega<\infty$ and let $\theta_{i} \in\{1,-1\}, 1 \leq i \leq n$, be fixed. Assume that (3.3)-(3.11) and (3.60) hold for each $1 \leq i \leq n$, and (3.71) holds. Let (3.72) be satisfied for $r=r_{\ell}, \ell=1,2, \ldots, k$, and (3.73) be satisfied for $R=R_{\ell}, \ell=1,2, \ldots, m$.

(a) If $m=k+1$ and $0<R_{1}<r_{1}<\cdots<R_{k}<r_{k}<R_{k+1}$, then (MI) has (at least) $2 k$ constant-sign solutions $u^{1}, \ldots, u^{2 k} \in\left(A_{\omega}(\mathbb{R})\right)^{n}$ such that

$$
0<R_{1}<\left|u^{1}\right|_{\omega} \leq r_{1} \leq\left|u^{2}\right|_{\omega}<R_{2}<\cdots \leq r_{k} \leq\left|u^{2 k}\right|_{\omega}<R_{k+1} .
$$

(b) If $m=k$ and $0<R_{1}<r_{1}<\cdots<R_{k}<r_{k}$, then (M1) has (at least) $2 k-1$ constant-sign solutions $u^{1}, \ldots, u^{2 k-1} \in\left(A_{\omega}(\mathbb{R})\right)^{n}$ such that

$$
0<R_{1}<\left|u^{1}\right|_{\omega} \leq r_{1} \leq\left|u^{2}\right|_{\omega}<R_{2}<\cdots<R_{k}<\left|u^{2 k-1}\right|_{\omega} \leq r_{k} .
$$

(c) If $k=m+1$ and $0<r_{1}<R_{1}<\cdots<r_{m}<R_{m}<r_{m+1}$, then (M1) has (at least $) 2 m$ constant-sign solutions $u^{1}, \ldots, u^{2 m} \in\left(A_{\omega}(\mathbb{R})\right)^{n}$ such that

$$
0<r_{1} \leq\left|u^{1}\right|_{\omega}<R_{1}<\left|u^{2}\right|_{\omega} \leq r_{2} \leq \cdots<R_{m}<\left|u^{2 m}\right|_{\omega} \leq r_{m+1} .
$$


(d) If $k=m$ and $0<r_{1}<R_{1}<\cdots<r_{k}<R_{k}$, then (M1) has (at least) $2 k-1$ constant-sign solutions $u^{1}, \ldots, u^{2 k-1} \in\left(A_{\omega}(\mathbb{R})\right)^{n}$ such that

$$
0<r_{1} \leq\left|u^{\prime}\right|_{\omega}<R_{1}<\left|u^{2}\right|_{\omega} \leq r_{2} \leq \cdots \leq r_{k} \leq\left|u^{2 k-1}\right|_{\omega}<R_{k} .
$$

EXAMPLE 2. Consider the nonlinear system of integral equations

$$
u_{i}(t)=\int_{t-\tau}^{t} g_{i}(t, s) \theta_{i} h_{i}(s)\left[\left|u_{1}(s)\right|^{\gamma}+\cdots+\left|u_{n}(s)\right|^{\gamma}+\left|u_{j}(s)\right|^{\delta}\right] d s,
$$

$t \in \mathbb{R}, 1 \leq i \leq n$; where $\gamma>0,0<\delta<1, j \in\{1,2, \ldots, n\}$ and $\theta_{i} \in\{-1,1\}$, $1 \leq i \leq n$, are fixed. For each $1 \leq i \leq n$, assume (3.3)-(3.7) and (3.48) hold, and also

$$
\inf _{t \in[0, \omega]} \int_{t-\tau}^{t} g_{j}(t, s) h_{j}(s) d s>0 .
$$

Then (3.81) has at least one constant-sign solution $u \in\left(A_{\omega}(\mathbb{R})\right)^{n}$ such that

$$
\min \{r, R\} \leq|u|_{\omega} \leq \max \{r, R\} \text { and }|u|_{\omega} \neq R,
$$

where $r$ and $R$ are positive numbers satisfying

$$
r<\left\{\inf _{t \in[0 . \omega]} \int_{t-\tau}^{t} g_{j}(t, s) h_{j}(s) d s\right\}^{1 /(1-\delta)}
$$

and

$$
n R^{\gamma-1}+R^{\delta-1}<\left(\max _{1 \leq i \leq n} K_{1, i}\right)^{-1}
$$

with

$$
K_{1, i}=\sup _{t \in[0, \omega]} \int_{t-\tau}^{t} g_{i}(t, s) h_{i}(s) d s, \quad 1 \leq i \leq n .
$$

To see that the above is true, we shall apply Theorem 3.7 with

$$
\left\{\begin{array}{l}
f_{i}(t, u)=\theta_{i} h_{i}(t)\left[\left|u_{1}\right|^{\gamma}+\cdots+\left|u_{n}\right|^{\gamma}+\left|u_{j}\right|^{\delta}\right], \\
b_{i}=h_{i}, \quad \psi_{i}(u)=\left|u_{1}\right|^{\gamma}+\cdots+\left|u_{n}\right|^{\gamma}+\left|u_{j}\right|^{\delta}, \quad 1 \leq i \leq n \\
\phi_{j}(u)=\left|u_{j}\right|^{\delta} .
\end{array}\right.
$$

Note that (3.8)-(3.11) and (3.60) are clearly satisfied. Next, in view of (3.82) and (3.87), condition (3.71) is satisfied with the fixed $j$ in (3.81). For this fixed $j$, it is obvious that $\phi_{j}(u) /\left|u_{j}\right|=1 /\left|u_{j}\right|^{1-\delta}$ is nonincreasing. Moreover, the inequality $r<\phi_{j}(r, \ldots, r) K_{2, j}$ in (3.72) reduces to

$$
\frac{r}{\phi_{j}(r, r, \ldots, r)}=r^{1-\delta}<K_{2, j}=\inf _{t \in[0, \omega]} \int_{t-\tau}^{t} g_{j}(t, s) h_{j}(s) d s
$$


which is equivalent to (3.84). Hence condition (3.72) is fulfilled. Finally, the inequality in (3.73) is reduced to $R>\left(n R^{\gamma}+R^{\delta}\right)\left(\max _{1 \leq i \leq n} K_{1, i}\right)$ which leads to (3.85).

It now follows from Theorem 3.7 that system (3.81) has at least one constant-sign solution $u \in\left(A_{\omega}(\mathbb{R})\right)^{n}$ satisfying (3.83)-(3.86).

Our next result also employs Leggett and Williams' fixed point theorem (Theorem 2.4).

THEOREM 3.11. Let $1 \leq p \leq \infty$, $q$ be such that $1 / p+1 / q=1,0<\omega<\infty$ and let $\theta_{i} \in\{1,-1\}, 1 \leq i \leq n$, be fixed. Assume that (3.3)-(3.11) and (3.60) hold for each $1 \leq i \leq n$. Moreover, suppose

$\left\{\begin{array}{l}\text { there exists some } j \in\{1,2, \ldots, n\} \text { such that } g_{j}(t, s) \geq a_{j}(s) \text { for all } \\ t \in[0, \omega] \text { and a.e. } s \in[0, \omega], \text { where } a_{j} \in L^{q}[0, \omega], a_{j} \text { is nonnegative, } \\ \text { and } a_{j}(t+\omega)=a_{j}(t) \text { for a.e. } t \in \mathbb{R},\end{array}\right.$

(for the same $j$ as in (3.88), there exists $r>0$ and a continuous function $\phi_{j}: \prod_{i=1}^{n}[0, \infty)_{i} \rightarrow[0, \infty)$; where $\phi_{j}\left(u_{1}, \ldots, u_{n}\right) /\left|u_{j}\right|$ $\{$ is 'nonincreasing' in the sense that for each $1 \leq k \leq n$, if $0<\left|u_{k}\right| \leq\left|v_{k}\right| \leq r$, then $\phi_{j}\left(u_{1}, \ldots, u_{n}\right) /\left|u_{j}\right| \geq \phi_{j}\left(v_{1}, \ldots, v_{n}\right) /\left|v_{j}\right|$. with $b_{j}(t) \phi_{j}(u) \leq \theta_{j} f_{j}(t, u)$ for a.e. $t \in[0, \omega]$ and all $u \in K$,

$\left\{\begin{array}{l}\text { for the same } j \text { and } r \text { as in (3.89), we have } \\ \qquad\left(\frac{\phi_{j}(r, \ldots, r)}{r}\right)^{N}\left(\prod_{i=1}^{N} \int_{l_{i}} a_{j}(s) b_{j}(s) d s\right)>1,\end{array}\right.$

where $N$ is the smallest positive integer such that $\omega / N \leq \tau / 2$ and
$I_{i}=[(i-1) \omega / N, i \omega / N], i=0,1, \ldots, N$,

and also (3.73) holds. Then (M1) has at least one constant-sign solution $u \in\left(A_{\omega}(\mathbb{R})\right)^{n}$ with $\min \{r, R\} \leq|u|_{\omega} \leq \max \{r, R\}$ and $|u|_{\omega} \neq R$.

Proof. Let $B, C$ and $u_{0}$ be defined as in the proof of Theorem 3.7. Then the same arguments give (3.75).

Next, we shall show that (3.77) is true. Let $u \in \partial \bar{C}_{r} \cap C\left(u_{0}\right)$. Then (3.78) follows. Further, from (3.89) we obtain (3.79) for $u \in C, t \in[0, \omega]$, a.e. $s \in[t-\tau, t]$ and some $j \in\{1, \ldots, n\}$ (the same $j$ as in (3.88)). Thus for $t \in[0, \omega]$ and the same $j$ as in (3.88), using (3.78), (3.79), (3.88) and (3.89) we find

$$
\begin{aligned}
\theta_{j} S_{j} u(t) & \geq \int_{t-\tau}^{t} g_{j}(t, s) b_{j}(s) \phi_{j}(u(s)) d s \\
& \geq \int_{t-\tau}^{t} a_{j}(s) b_{j}(s) \frac{\phi_{j}(u(s))}{\theta_{j} u_{j}(s)} \theta_{j} u_{j}(s) d s
\end{aligned}
$$




$$
\geq \frac{\phi_{j}(r, \ldots, r)}{r} \int_{t-\tau}^{t} a_{j}(s) b_{j}(s) \theta_{j} u_{j}(s) d s .
$$

We claim that there exists a $t_{0, j} \in[0, \omega]$ with

$$
\frac{\phi_{j}(r, \ldots, r)}{r} \int_{t_{0 . j}-\tau}^{t_{0, j}} a_{j}(s) b_{j}(s) \theta_{j} u_{j}(s) d s>\theta_{j} u\left(t_{0, j}\right)
$$

If our claim is true, then it follows from (3.91) that

$$
\theta_{j} S_{j} u\left(t_{0, j}\right) \geq \frac{\phi_{j}(r, \ldots, r)}{r} \int_{t_{0 . j}-\tau}^{t_{0 . j}} a_{j}(s) b_{j}(s) \theta_{j} u_{j}(s) d s>\theta_{j} u\left(t_{0, j}\right)
$$

and therefore (3.77) holds. Using a similar argument as in the proof of Theorem 3.7 , we then apply Theorem 2.4 to obtain the result.

It remains to prove our claim. Suppose (3.92) is false. Then

$$
\theta_{j} u_{j}(t) \geq \frac{\phi_{j}(r, r, \ldots, r)}{r} \int_{t-\tau}^{t} a_{j}(s) b_{j}(s) \theta_{j} u_{j}(s) d s \quad \text { for all } t \in[0, \omega] .
$$

Note that if $t \in I_{i}$ for some $i \in\{1, \ldots, N\}$, then $I_{i-1} \subseteq[t-\tau, t]$ since $\omega / N \leq \tau / 2$. This together with (3.93) gives for $i \in\{1, \ldots, N\}$,

$$
\begin{aligned}
\int_{l_{i}} a_{j}(t) b_{j}(t) \theta_{j} u_{j}(t) d t \\
\quad \geq \frac{\phi_{j}(r, \ldots, r)}{r} \int_{l_{i}} a_{j}(t) b_{j}(t) \int_{t-\tau}^{t} a_{j}(s) b_{j}(s) \theta_{j} u_{j}(s) d s d t \\
\quad \geq \frac{\phi_{j}(r, \ldots, r)}{r} \int_{l_{i}} a_{j}(t) b_{j}(t) \int_{l_{i-1}} a_{j}(s) b_{j}(s) \theta_{j} u_{j}(s) d s d t \\
\quad=\frac{\phi_{j}(r, \ldots, r)}{r}\left(\int_{l_{i}} a_{j}(t) b_{j}(t) d t\right)\left(\int_{l_{i-1}} a_{j}(s) b_{j}(s) \theta_{j} u_{j}(s) d s\right) .
\end{aligned}
$$

Applying the above repeatedly yields

$$
\begin{aligned}
\int_{I_{N}} a_{j}(t) b_{j}(t) \theta_{j} u_{j}(t) d t \\
\geq\left(\frac{\phi_{j}(r, \ldots, r)}{r}\right)^{N}\left(\prod_{i=1}^{N} \int_{l_{i}} a_{j}(t) b_{j}(t) d t\right)\left(\int_{l_{0}} a_{j}(s) b_{j}(s) \theta_{j} u_{j}(s) d s\right) \\
=\left(\frac{\phi_{j}(r, \ldots, r)}{r}\right)^{N}\left(\prod_{i=1}^{N} \int_{I_{i}} a_{j}(t) b_{j}(t) d t\right)\left(\int_{I_{N}} a_{j}(s) b_{j}(s) \theta_{j} u_{j}(s) d s\right) .
\end{aligned}
$$


If $\int_{I_{N}} a_{j}(t) b_{j}(t) \theta_{j} u_{j}(t) d t>0$, then (3.94) leads to

$$
\left(\frac{\phi_{j}(r, \ldots, r)}{r}\right)^{N}\left(\prod_{i=1}^{N} \int_{t_{i}} a_{j}(t) b_{j}(t) d t\right) \leq 1,
$$

which contradicts (3.90). On the other hand, if $\int_{I_{N}} a_{j}(t) b_{j}(t) \theta_{j} u_{j}(t) d t=0$, then since $\theta_{j} u_{j}(t)>0$ for $t \in[0, \omega]$, we must have $a_{j}(t) b_{j}(t)=0$ for a.e. $t \in I_{N}$. This again contradicts (3.90). Hence we have shown that our claim (3.92) is true. The proof is now complete.

REMARK 3.12. Remark 3.8 also holds for Theorem 3.11 .

REMARK 3.13. Theorem 3.11 extends the results obtained in [15].

Our final result generalises Theorem 3.11 to give the existence of multiple constantsign periodic solutions.

THEOREM 3.14. Let $1 \leq p \leq \infty, q$ be such that $1 / p+1 / q=1,0<\omega<\infty$ and let $\theta_{i} \in\{1,-1\}, 1 \leq i \leq n$, be fixed. Assume that (3.3)-(3.11) and (3.60) hold for each $1 \leq i \leq n$, and (3.88) holds. Let (3.89) and (3.90) be satisfied for $r=r_{\ell}, \ell=1,2, \ldots, k$, and (3.73) be satisfied for $R=R_{\ell}, \ell=1,2, \ldots, m$. Then conclusions (a)-(d) of Theorem 3.10 hold.

REMARK 3.15. Similar to Remark 3.2, in Theorems 3.3-3.14 we can replace conditions (3.3), (3.4), (3.6)-(3.8) and (3.11) with (3.43)-(3.46).

\section{References}

[1] R. P. Agarwal, D. O'Regan and P. J. Y. Wong, Positive solutions of differential, difference and integral equations (Kluwer, Dordrecht, 1999).

[2] R. P. Agarwal, D. O'Regan and P. J. Y. Wong, "Constant-sign $L^{p}$ solutions for a system of integral equations", Results Math. 46 (2004) 195-219.

[3] R. P. Agarwal, D. O'Regan and P. J. Y. Wong, "Constant-sign solutions of a system of Fredholm integral equations", Acta Appl. Math. 80 (2004) 57-94.

[4] R. P. Agarwal, D. O'Regan and P. J. Y. Wong, "Eigenvalues of a system of Fredholm integral equations", Math. Comput. Modelling 39 (2004) 1113-1150.

[5] R. P. Agarwal, D. O'Regan and P. J. Y. Wong, "Triple solutions of constant sign for a system of Fredholm integral equations", Cubo 6 (2004) $1-45$.

[6] R. P. Agarwal, D. O'Regan and P. J. Y. Wong, "Constant-sign solutions of a system of integral equations: The semipositone and singular case", Asymptot. Anal. 43 (2005) 47-74.

[7] R. P. Agarwal, D. O' Regan and P. J. Y. Wong. "Constant-sign periodic and almost periodic solutions for a system of integral equations", Acta Appl. Math. (to appear). 
[8] R. P. Agarwal and P. J. Y. Wong, Advanced topics in difference equations (Kluwer, Dordrecht, 1997).

[9] K. L. Cooke and J. L. Kaplan, "A periodicity threshold theorem for epidemics and population growth", Mat. Biosc. 31 (1976) 87-104.

[10] L. H. Erbe, S. Hu and H. Wang, "Multiple positive solutions of some boundary value problems", J. Math. Anal. Appl. 184 (1994) 640-648.

[11] L. H. Erbe and H. Wang, "On the existence of positive solutions of ordinary differential equations", Proc. Amer. Math. Soc. 120 (1994) 743-748.

[12] D. Guo and V. Lakshmikantham, Nonlinear problems in abstract cones (Academic Press, San Diego, 1988).

[13] M. A. Krasnosel'skii, Positive solutions of operator equations (Noordhoff, Groningen, 1964).

[14] R. W. Leggett and L. R. Williams, "Multiple positive fixed points of nonlinear operators on ordered Banach spaces", Indiana Univ. Math. J. 28 (1979) 673-688.

[15] R. W. Leggett and L. R. Williams, "A fixed point theorem with application to an infectious disease model", J. Math. Anal. Appl. 76 (1980) 91-97.

[16] W. Lian, F. Wong and C. Yeh, "On the existence of positive solutions of nonlinear second order differential equations", Proc. Amer. Math. Soc. 124 (1996) 1117-1126.

[17] R. Nussbaum, "A periodicity threshold theorem for some nonlinear integral equations", SIAM J. Anal. 9 (1978) 356-376.

[18] D. O'Regan and M. Meehan, Existence theory for nonlinear integral and integrodifferential equations (Kluwer, Dordrecht, 1998).

[19] H. Smith, "On periodic solutions of delay integral equations modelling epidemics and population growth", Ph. D. Thesis, University of Iowa City, 1976.

[20] L. R. Williams and R. W. Leggett, "Nonzero solutions of nonlinear integral equations modeling infectious disease", SIAM J. Anal. 13 (1982) 112-121. 